\title{
Reaction mechanisms in heavy ion fusion
}

\author{
L. F. Canto ${ }^{1, \text { a }}$, P. R. S. Gomes ${ }^{2}$, J. Lubian ${ }^{2}$, and M. S. Hussein ${ }^{3}$ \\ 1 Instituto de Física, Universidade Federal do Rio de Janeiro, CP 68528, Rio de Janeiro, R.J., 21941-972, Brazil \\ 2 Instituto de Física, Universidade Federal Fluminense, Av. Litorânea s/n, 24210-340 Niteroi, R.J. , Brazil \\ 3 Instituto de Física, Universidade de São Paulo, CP 66318, 05389-970, São Paulo, SP, Brazil
}

\begin{abstract}
We discuss the reaction mechanisms involved in heavy ion fusion. We begin with collisions of tightly bound systems, considering three energy regimes: energies above the Coulomb barrier, energies just below the barrier and deep sub-barrier energies. We show that channel coupling effects may influence the fusion process at above-barrier energies, increasing or reducing the cross section predicted by single barrier penetration model. Below the Coulomb barrier, it enhances the cross section, and this effect increases with the system's size. It is argued that this behavior can be traced back to the increasing importance of Coulomb coupling with the charge of the collision partners. The sharp drop of the fusion cross section observed at deep sub-barrier energies is addressed and the theoretical approaches to this phenomenon are discussed. We then consider the reaction mechanisms involved in fusion reactions of weakly bound systems, paying particular attention to the calculations of complete and incomplete fusion available in the literature.
\end{abstract}

\section{Introduction}

Heavy ion fusion is a subject that has received a great amount of attention in the last four decades, but this field is still quite alive and periodically new phenomena are discovered. During the 1960s and 1970s, most of the investigation in this field was concentrated in the energy regime just above Coulomb barrier, the so-called "Region I". In this regime, fusion is the main reaction channel and its cross section has a geometrical nature. It has been frequently described as the penetration through a single radial barrier (SBPM), with the transmission factors given by the classical approximation. At higher energies, the so-called "Region II", other reaction processes compete with fusion, such as deep inelastic collisions and projectile breakup followed by incomplete fusion. A widely used model that describes the fusion process in both regions was proposed by Glas and Mosel [1]. Later, in the late 1970s and 1980s, the theoretical and experimental efforts were mainly concentrated in the energy region below the Coulomb barrier, where tunneling takes place and the fusion process is strongly influenced by the nuclear structure of the collision partners. Several experiments found cross sections strongly enhanced as compared with SBPM results, where the intrinsic degrees of freedom are neglected. Thus, in this energy regime the fusion cross section should be calculated by the Coupled channel method.

More recently, the development of more sophisticated experimental techniques lead to the discovery of new phenomena. One of them is the steep fall off of the fusion

\footnotetext{
a e-mail: canto@if.ufrj.br
}

cross section in so far unexplored energy regime: deep subbarrier energies. This regime is very important to understand reactions that occur in nucleosynthesis. Another very rich field is the production of super-heavy elements (SHE). For very massive systems there is a strong inhibition of the fusion process by the quasi-fission mechanism. Finally, with the availability of radioactive beams, a new research field was developed: reactions involving weakly bound nuclei. These reactions are influenced by lower barriers arising from weakly bound nucleons and strong couplings with channels in the continuum, corresponding to the breakup of one of the collision partners. In this talk we will discuss the research going on in some of these fields. Owing to the limitation of time, we will have to leave out many interesting topics.

\section{Basic theoretical ideas}

The simplest theoretical approach for fusion reactions is to neglect the influence of intrinsic degrees of freedom of the collision partners and treat the collision as a potential scattering problem with a complex potential. Owing to the imaginary part, the stationary continuity equation is violated. Proceeding as in the derivation of this equation one finds that the fusion cross section is given by

$$
\sigma_{\mathrm{F}}=\frac{k}{E}\langle\psi|(-W)| \psi\rangle,
$$

$\psi$ is the scattering wave function with energy $E$ and wave number $k$, and $W$ is the imaginary part of the potential. 
Frequently, the intrinsic degrees of freedom have strong influence on the fusion cross section. Thus, they must be taken into account. This can be done through the coupled channel (CC) method. In this case, the fusion cross section becomes

$$
\sigma_{\mathrm{F}}=\frac{k}{E}\langle\Psi|(-W)| \Psi\rangle .
$$

Above, $\Psi$ stands for the many-body wave function obtained from the CC equations, and $W$ is the non-hermitian part of the now potential operator, which now depends also on the intrinsic degrees of freedom. In some calculations the imaginary part of the interaction is diagonal in channel space. It is then possible to write the cross section as the sum of separate contributions from each channel included in the calculation, as

$$
\sigma_{\mathrm{F}}^{\mathrm{CC}}=\sum_{\alpha} \sigma_{\mathrm{F}}^{(\alpha)} \text {, with } \sigma_{\mathrm{F}}^{(\alpha)}=\frac{k}{E}\left\langle\psi_{\alpha}\left|\left(-W_{\alpha, \alpha}\right)\right| \psi_{\alpha}\right\rangle \text {. }
$$

Above, $\psi_{\alpha}$ is the relative wave functions in channel- $\alpha$ and $W_{\alpha, \alpha}$ is the corresponding diagonal matrix-element of the non-hermitian part of the interaction.

The first authors to investigate channel coupling effects on fusion were Dasso, Landowne and Winther [2,3] and Lindsay and Rowley [4]. In their simplified treatment of the CC problem, they found that channel coupling leads to large enhancements of the fusion cross section at subbarrier energies. This finding was confirmed by more realistic calculations. As an example, we show in figure 1 fusion cross sections for the ${ }^{16} \mathrm{O}+{ }^{154} \mathrm{Sm}$ system, obtained in different calculations. In this case there are strong couplings of the elastic channel and inelastic channels associated with the rotational states of the highly deformed ${ }^{154} \mathrm{Sm}$ nucleus. The figure shows the optical model cross section (dotted line), where channel couplings are neglected, together with coupled channel cross sections. The dashed line corresponds to fusion in the elastic channel (that is, the first term in Eq. (2)) and the solid line is the sum of contributions from all channels. One notices that the cross sections obtained by the CC calculations $\left(\sigma_{\mathrm{F}}^{(0)}\right.$ and $\left.\sigma_{\mathrm{F}}^{\mathrm{CC}}\right)$ are two orders of magnitude larger then the one without couplings $\left(\sigma_{\mathrm{F}}^{\mathrm{opt}}\right)$. The difference between the two $\mathrm{CC}$ calculations: $\sigma_{\mathrm{F}}^{\mathrm{CC}}-\sigma_{\mathrm{F}}^{(0)}$, which corresponds to absorption in inelastic channels, is quite relevant.

\section{Fusion of tightly bound systems}

We consider here collisions of tightly bound systems in three energy regimes. Collisions involving weakly bound nuclei are influenced by additional reaction and will be considered separately.

\subsection{Above-barrier energies}

We begin with collisions at energies a few $\mathrm{MeV}$ above the Coulomb barrier. At higher energies, the fusion cross section is affected by other processes, which we will not discuss in the present work. Usually, the quantum mechanical

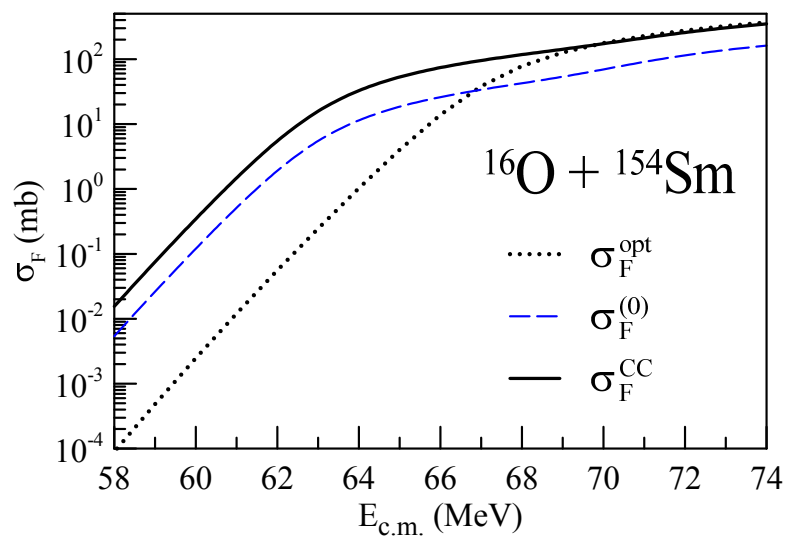

Fig. 1. Different calculations of the fusion cross section for the ${ }^{16} \mathrm{O}+{ }^{154} \mathrm{Sm}$ system. For details, see the text.

description of scattering uses the partial-wave expansion

$$
\sigma_{\mathrm{F}}=\frac{\pi}{k^{2}} \sum_{l}(2 l+1) P_{l},
$$

where $P_{l}$ is the fusion probability at the $l^{\text {th }}$-partial wave. Frequently, one approximates $P_{l}$ by the probability, $T_{l}$, of tunneling through the $l$-dependent barrier given by the sum of the the optical, the Coulomb and the centrifugal potentials. In this energy regime, one can use the classical approximation for $T_{l}$. It is 1 if the collision energy is larger than the barrier height, $V_{\mathrm{B}}(l)$, and 0 otherwise. With this assumption, the partial wave series can be summed analytically and the result is

$$
\sigma_{\mathrm{F}} \simeq \pi R_{B}^{2}\left[1-\frac{V_{\mathrm{B}}}{E}\right],
$$

where $V_{\mathrm{B}}$ is the height of the $l=0$ barrier and $R_{\mathrm{B}}$ is the corresponding barrier radius. According to this equation, the cross section at above-barrier energies is a straight line in terms of the variable $1 / E$. Indeed, this behavior can be observed for many systems and many authors used it to extract the parameters of the fusion barrier. This could be a useful method to extract information about the Coulomb barrier and the bare nuclear potential. Then, comparing the experimental cross section at sub-barrier energies with the SBPM cross section obtained with this potential, one could estimate the enhancement of the cross section in this energy regime. However, this procedure has a shortcoming: it assumes that channel coupling effects can be neglected above the Coulomb barrier. Otherwise, the barrier parameters extracted by this method would correspond to some combination of the optical and a polarization potential simulating channel coupling effects.

This assumption can be checked comparing SBPM results, obtained with a standard folding potential, with the corresponding experimental data. In order to eliminate trivial differences among cross sections for different systems, we use reduced experimental data throughout this work. 

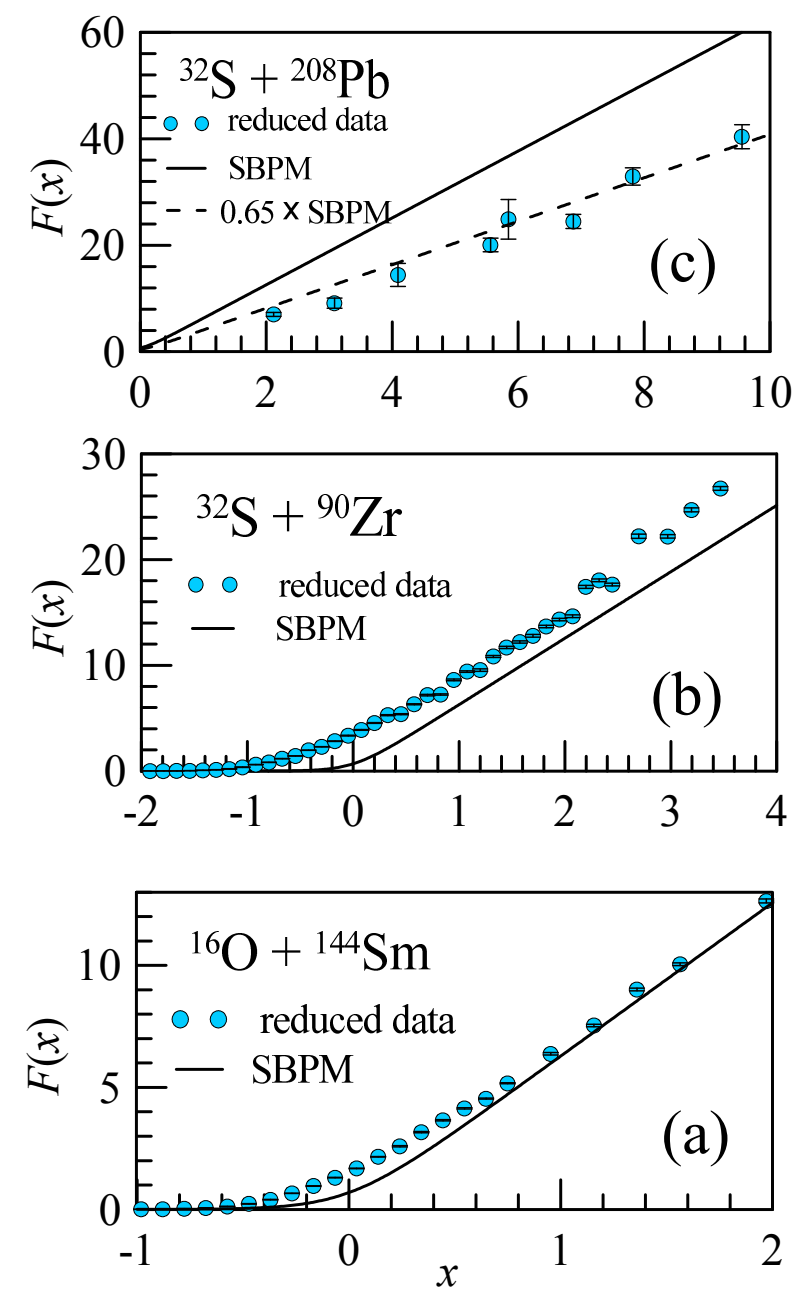

Fig. 2. Comparison of the predictions of folding potentials[8] with experimental data for a few tightly bound systems. The data in panels (a), (b) and (c) are respectively from Refs. [9], [10] and [11].

Our prescription to reduce the cross sections and the collision energies is to transform to the dimensionless quantities [5,6]: $E \rightarrow x=\left(E-V_{\mathrm{B}}\right) / \hbar \omega$ and $\sigma_{\mathrm{F}} \rightarrow F(x)=$ $\sigma_{\mathrm{F}} \times 2 E / \hbar \omega R_{\mathrm{B}}^{2}$, where $\hbar \omega$ is the barrier curvature parameter. For cross sections that are not affected by channel coupling and can be approximated by Wong's formula[7], the reduction leads to the system-independent function of $x$,

$$
F_{0}(x)=\ln [1+\exp (2 \pi x)]
$$

which is called universal fusion function (UFF).

In figure 2, we show the SBPM and the experimental cross sections for a few systems. The comparisons indicate that CC effects may be negligible for some systems (figure 2(a)) but they may have appreciable influence on the cross section. It can lead to some enhancement (figure 2 (b)) or to some suppression (figure 2(c)). Note that in this example the suppression similar to that found for some weakly bound projectile on heavy targets, discussed in a forthcoming section. We conclude that extracting parameters of the

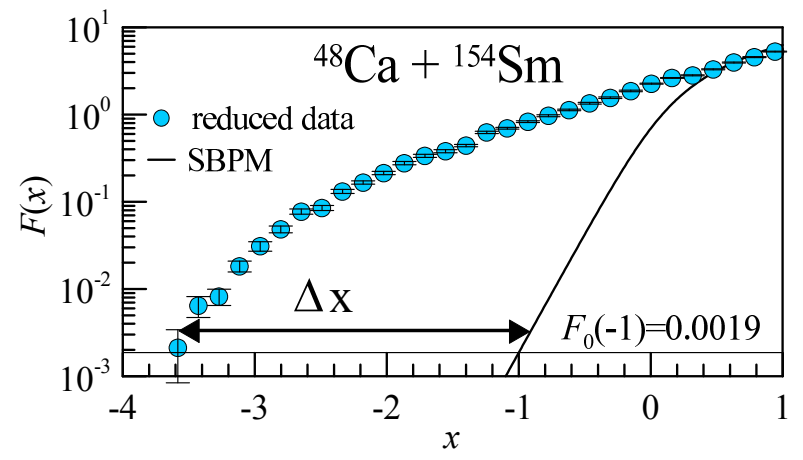

Fig. 3. The shift on the $x$ variable as a measure of the enhancement of $\sigma_{\mathrm{F}}$.

optical potential from above barrier data may be an unreliable method.

\subsection{Fusion at moderate sub-barrier energies}

Now we consider the cross section a few $\mathrm{MeV}$ below the Coulomb barrier. We investigate the enhancement in the fusion cross section as a function of the system size.

We measure the enhancement of the reduced fusion cross section by the shift, $\Delta x$, that make it equal to the UFF. Since frequently $F(x)$ and $F_{0}(x)$ have different slopes, we take as a reference the value $x=-1$, where the UFF has the value $F_{0}(x=-1)=0.0019$. This procedure is illustrated in figure 3 , in the case of the ${ }^{48} \mathrm{Ca}+{ }^{154} \mathrm{Sm}$ (the data are from Ref. [12]. We investigate the dependence of the shift on the system mass, represented by the size parameter,

$$
\zeta=\frac{Z_{P} Z_{T}}{A_{P}^{1 / 3} A_{T}^{1 / 3}\left(A_{P}^{1 / 3}+A_{P}^{1 / 3}\right)} .
$$

Above, $Z_{P}$ and $A_{P}\left(Z_{T}\right.$ and $\left.A_{T}\right)$ are respectively the atomic and mass numbers of the projectile (target). The results for several systems are represented by small symbols in figure 4. The data are from Refs. [12-17, 10, 18,9]. The enhancement has a clear tendency of growing with the system size, although there are fluctuations around the average behavior, associated the specific nuclear properties of the system. This trend has been associated with the growing importance of the neck degree of freedom [19]. However, it may have a simpler explanation. Since channels associated with the Coulomb excitation of collective channels are known to have a large contribution to the enhancement of $\sigma_{\mathrm{F}}$, this trend may simply result from the fact that the strength of the coupling grows with the system's charge, it. This possibility can be checked by renormalizing the fusion cross section by the factor $\sigma_{\mathrm{F}}^{\mathrm{opt}} / \sigma_{\mathrm{F}}^{\mathrm{CC}}$, where $\sigma_{\mathrm{F}}^{\mathrm{CC}}$ is the cross section obtained with a $\mathrm{CC}$ calculation including the channels associated with the relevant collective excitations and $\sigma_{\mathrm{F}}^{\mathrm{opt}}$ is the optical model cross section (with the couplings switched off). If the effect results from these couplings, it should not remain when $\Delta x$ determined from the renormalized cross section. We have checked this point for a few of 


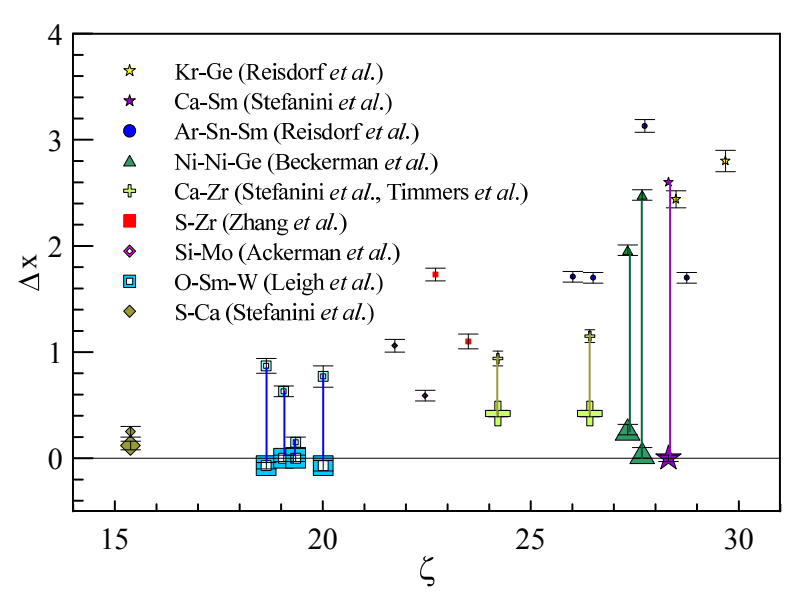

Fig. 4. Size dependence of the shift $\Delta x$ variable as a measure of the enhancement of $\sigma_{\mathrm{F}}$.
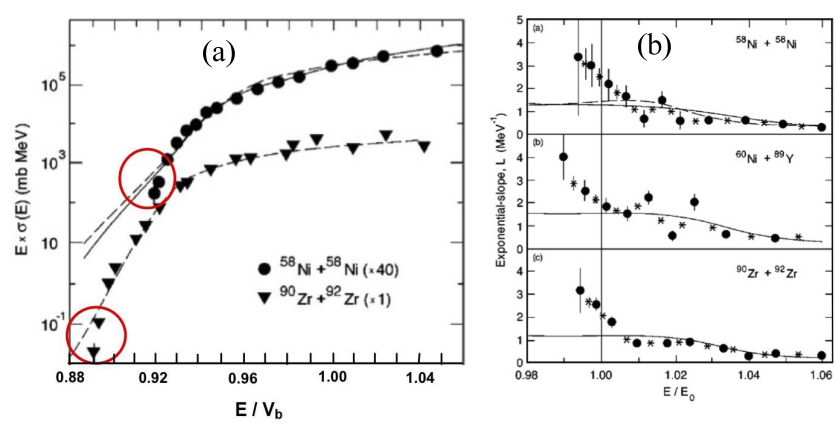

Fig. 5. Fusion reactions at deep sub-barrier energies. Panel (a) shows the cross sections vs. the collision energy. In panel (b) the logarithmic derivative of $E \sigma_{\mathrm{F}}$ (labeled by $L$ ) is plotted against $E$.

the systems considered in the figure. The results for these systems are represented by the same symbols with larger sizes. The enhancement comes down to small values and the growing trend with the size parameters disappears.

\subsection{Fusion at deep sub-barrier energies}

An unexpected phenomenon was observed in the study of fusion reactions at deep sub-barrier energies. This became possible with the possibility of measuring very small cross section (tens of nanobarns) with high accuracy. It was found that below some energy $E_{0}$ (indicated by circles in figure 5 (a)), which depends on the system, the cross section decreases much faster than expected. This phenomenon can be observed more clearly when one plots the logarithmic derivative of the product $E \sigma_{\mathrm{F}}$, as a function of energy. If the cross section could be approximated by the Wong's formula, this quantity would converge to a constant value as the energy decreases well below the Coulomb barrier. The hindrance of the fusion cross section at deep sub-barrier energies was first observed for the ${ }^{58} \mathrm{Ni}+{ }^{58} \mathrm{Ni}$ system [20, 21] (see figure 5) but similar results have been reported for several other systems [22-27]. It should be stressed

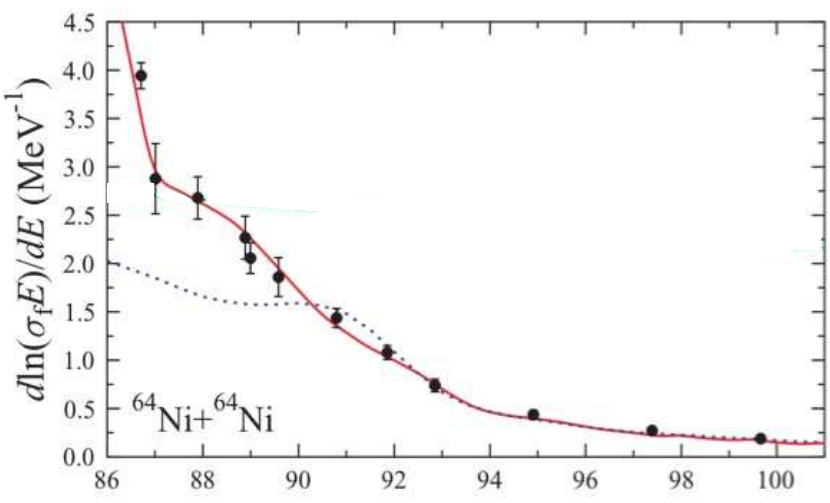

Fig. 6. The logarithmic derivative of $E \sigma_{\mathrm{F}}$ for the ${ }^{64} \mathrm{Ni}+{ }^{64} \mathrm{Ni}$ system. The CC calculation of Mişicu and Esbensen with the M3Y+repulsion is represented by the solid line while the dotted line is the CC calculation with the Akyüz-Winther potential. Further details are given in Ref. [28]

that the cross section is hindered with respect to the values predicted by CC calculations. However, in many instances the cross section remains larger than the prediction of the SBPM. In fact, the remarkable effect is the steeper fall off of the cross section at deep sub-barrier energies.

Understanding the origin of this phenomenon became a great challenge for theorists. Since coupled channel effects saturate at very low energies, the hindrance effect should be traced back to the bare nucleus-nucleus potential. Hagino, Rowley and Dasgupta showed that in order to reproduce the data with a Woods-Saxon (WS) potential, it should have a very large diffusivity parameter, which would not be consistent with scattering data. Thus, they argued that a WS shape was not appropriate to parametrize the nucleus-nucleus potential at all projectile-target distances. Dasso and Pollarolo [29] pointed out that the energy $E_{0}$, where the steeper fall off begins, corresponds to the dip in the nuclear+Coulomb potential. This indicated that the nucleus-nucleus potential should be shallower than those derived with the usual potential models.

It is clear that fusion data at deep sub-barrier energies provide relevant information about the nucleus-nucleus potential, which are not available in scattering experiments. Mişicu and Esbensen showed that the predictions of CC calculations using either the Akyüz-Winther [30] potential or a folding potential [31] with the M3Y interaction [32] would not reproduce the steep fall off observed in the fusion data for the ${ }^{64} \mathrm{Ni}+{ }^{64} \mathrm{Ni}$ system. However, they got good agreement with the data adding a repulsive term to the M3Y nucleon-nucleon interaction. The strength of this term was related to the incompressibility of nuclear matter, calculated by the Thomas-Fermi model. The results of this calculation (solid line) is compared with the data in figure 6. This model was equally successful for several other systems [28,33-35].

Ichikawa, Hagino and Iwamoto proposed a different mechanism to explain the phenomenon. They kept the frozen 


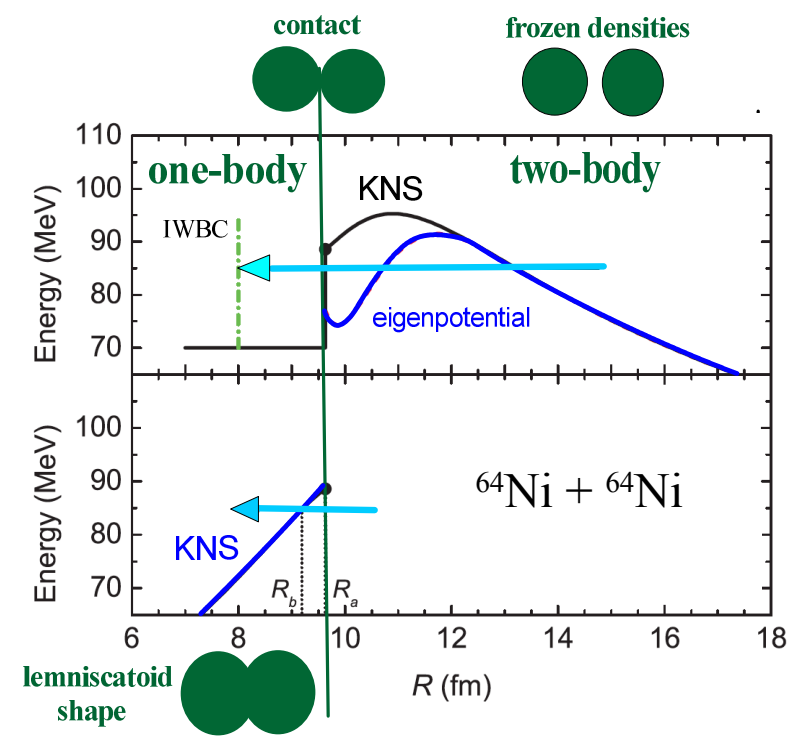

Fig. 7. The potential barriers in the two-body and one-body regimes, as described in the text. For details, see Ref. [35].

densities approximation for the two-body regime, where the projectile-target separation is larger than the contact radius, $R_{\text {cont }}$. This radius is defined by the condition that summed densities at the nuclear surfaces reaches the central nuclear density. However, for $r<R_{\text {cont }}$, they adopted a one-body regime, where the system shape evolves adiabatically with a lemniscatoid shape. The bare nuclear potential was given by the folding of the Krappe-Nix-Sierk interaction [36] with the nuclear densities, subtracting its value at asymptotic distances. The situation is depicted in figure 7. The upper panel shows the two-body regime representing both the bare potential and the eigen-potential arising from channel-coupling. The fusion process requires that the system tunnels through the eigen-potential. In addition, for energies below the bare potential at the contact radius, it is necessary to tunnel also through the inner barrier in the one-body regime, indicated in the bottom panel of figure 7. An essential ingredient of this approach is the neglect of channel couplings in the one-body regime. This is because the CC channel basis in not appropriate to describe the states of the system there. In this way, the model has a sharp transition between the two regimes. This drastic assumption is relaxed in a subsequent paper [37], where the transition is given by a smooth function of the projectiletarget distance. In Refs. [24,38], transitions of this kind are associated with decoherence.

\section{Fusion of weakly bound systems}

Fusion of weakly bound systems differs from fusion of tightly bound systems in two-ways [39]. First, their fusion barriers tends to be slightly lower and thicker, owing to the longer tail of the nuclear density. Second, they are influenced by the coupling with the breakup channel, which can be neglected in collisions of tightly bound systems at nearbarrier energies. One consequence of breakup coupling is that it gives rise to a new kind of fusion, which is called incomplete fusion (ICF). It corresponds to a two-step process: the projectile breaks up as it approaches the target and then one fragment is absorbed while the rest of the projectile's mass escapes the interaction region. In most experimental and theoretical studies, only the sum of $\mathrm{CF}$ and ICF - called total fusion (TF) - is determined. Separate measurements of $\sigma_{\mathrm{CF}}$ and $\sigma_{\mathrm{ICF}}$ is possible only for a few systems. Separate calculations of these quantities are also very hard to perform. These calculations are discussed below.

\subsection{Calculations of fusion cross sections}

The earliest calculations of fusion cross sections for weakly bound systems where performed for the exotic ${ }^{11} \mathrm{Li}$ projectiles. They were base on very crude approximations for the continuum and lead to opposing conclusion. Hussein et al. [40] and Takigawa et al. [41] took the effects of continuum coupling into account through the introductions of an imaginary polarization potential. Its real part was mocked up by a shift in the collision energy. They concluded that the breakup coupling hindered fusion. On the other hand Dasso and Vitturi [42] represented the continuum by a bound channel, leaving out its contribution to the $\mathrm{CF}$ cross section, and found that the coupling enhanced the CF cross section. Since these calculations made drastic assumptions about the nature of the breakup channel, it is not surprising that they lead to different conclusion. Nearly a decade later, separate calculations of CF and ICF cross sections were performed by a classical three-body (target+fragment1+fragment2) model $[43,44]$ in two dimensions. In this model, the collision dynamics transferred energy to the relative motion of the fragments and this could lead to breakup, CF and ICF. The predictions of the model at above-barrier energies were in reasonable agreement with the data for ${ }^{6,7} \mathrm{Li}+{ }^{209} \mathrm{Bi}$. A similar model in three dimensions and treating the breakup process stochastically was proposed by Diaz-Torres et al. [45,46].

The first realistic calculations of CF and ICF cross sections were performed by Hagino et al. [47], for the ${ }^{11} \mathrm{Be}$ $+{ }^{208} \mathrm{~Pb}$ system. They used the continuum discretized couple channel (CDCC) method [48], in which the continuum (breakup) is approximated by a finite set of channels. In their calculations, the real part of the projectile-target interaction was given by the sum of potentials between the target and each of the fragments $\left({ }^{10} \mathrm{Be}\right.$ and $\left.n\right)$. This interaction accounted for both the bare potential and the couplings. The fusion process resulted from a short-range imaginary potential depending on the projectile-target coordinates. In this case, the channel-coupling interaction is real, so that the contribution from each channel for the fusion cross section can be evaluated separately, according to Eq. (3). The CF cross section was then taken as the sum of contributions from the bound channels, whereas the ICF cross section was given by the channels in the continuum. It is important 
to remark that this procedure to evaluate $\mathrm{CF}$ and ICF is reasonable only when the absorbed fragment $\left({ }^{10} \mathrm{Be}\right)$ is much heavier than the other fragment (n). Two years later, DiazTorres and Thompson [49] performed a similar calculation for the same system. This calculation differed from the previous one in two ways. First, they used a larger continuum space. The second difference, which was much more important, was that they included couplings among continuum states (CCC), which where neglected by Hagino et al. [47]. These couplings turned out to be very important for both the TF and the CF cross sections. This is illustrated in figure 8. Panel (a) shows the TF and ICF cross section when continuum-continuum couplings are neglected. The TF cross section (long-dashed line) is enhanced with respect to the the optical model cross section (short-dashed line), where all couplings are switched off, in the whole energy region. On the other hand, the CF cross section is strongly enhanced at low energies and is hindered at high energies. The transition takes place at $E \simeq 40 \mathrm{MeV}$, that is a few $\mathrm{MeV}$ above the barrier $\left(V_{B}=36 \mathrm{MeV}\right)$. When CCC is taken into account (panel (b)) the situation changes drastically. Both cross sections become much smaller. Now the TF cross section falls below the optical model cross section at energies above the barrier. The effects on $\mathrm{CF}$ is much more pronounced. The transition from enhancement to hindrance takes place at $E \simeq 33 \mathrm{MeV}$, which is a few MeV below the barrier. Furthermore, the enhancement at low energies is much weaker than before. The reduction of fusion could arise either from an increase of absorption into the breakup channel or to a repulsive effect. These possibilities would lead to different consequences on the cross sections for other channels. Increased absorption would lead to larger breakup cross section and less elastic scattering. On the other hand, repulsion would increase the elastic cross section. This problem was investigated in calculations of breakup and elastic scattering for the ${ }^{8} \mathrm{~B}$ $+{ }^{58} \mathrm{Ni}$ system, with and without $\mathrm{CCC}$ [50]. The results are shown in figure 9. Comparing the cross sections calculated with (solid lines) and without (dashed lines) CCC, one concludes that the hindrance of the fusion cross sections arises from a repulsive effect. This was confirmed by direct calculation of the breakup polarization potentials for this system [50]. Inclusion of CCC makes the real potential less attractive and weakens its imaginary part.

Recently, Hashimoto et al. [51] proposed an interesting method to evaluate separately the CF and ICF cross sections in CDCC calculations. The projectile-target interaction is given by the sum of complex potentials between the target and each of the fragments. In this case, the imaginary parts give fusion of the target with each of the fragments. Several calculations where carried out with this kind of interaction, like the CDCC calculations of Refs. [52-55] and the time-dependent description of the three-body system [56]. The special feature of Ref. [51] is that it allows the calculation of CF and ICF separately. The cross section is expressed in terms of the many-body wave function, $\Psi(\mathbf{R}, \mathbf{r})$, where $\mathbf{R}, \mathbf{r}$ stand respectively for the projectiletarget and fragment-fragment separation vectors, through Eq. (2). Transforming to the fragments coordinates $\mathbf{R}, \mathbf{r} \rightarrow$
WITHOUT continuum-continuum couplings

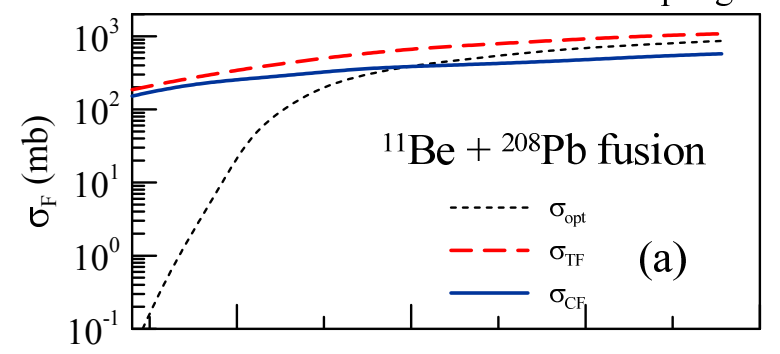

WITH continuum-continuum couplings

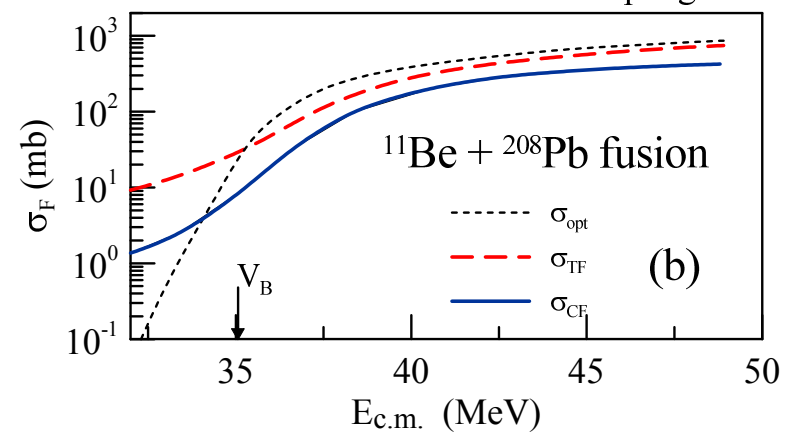

Fig. 8. CDCC calculations of CF and ICF cross sections for the ${ }^{11} \mathrm{Be}+{ }^{208} \mathrm{~Pb}$ system, with and without CCC.

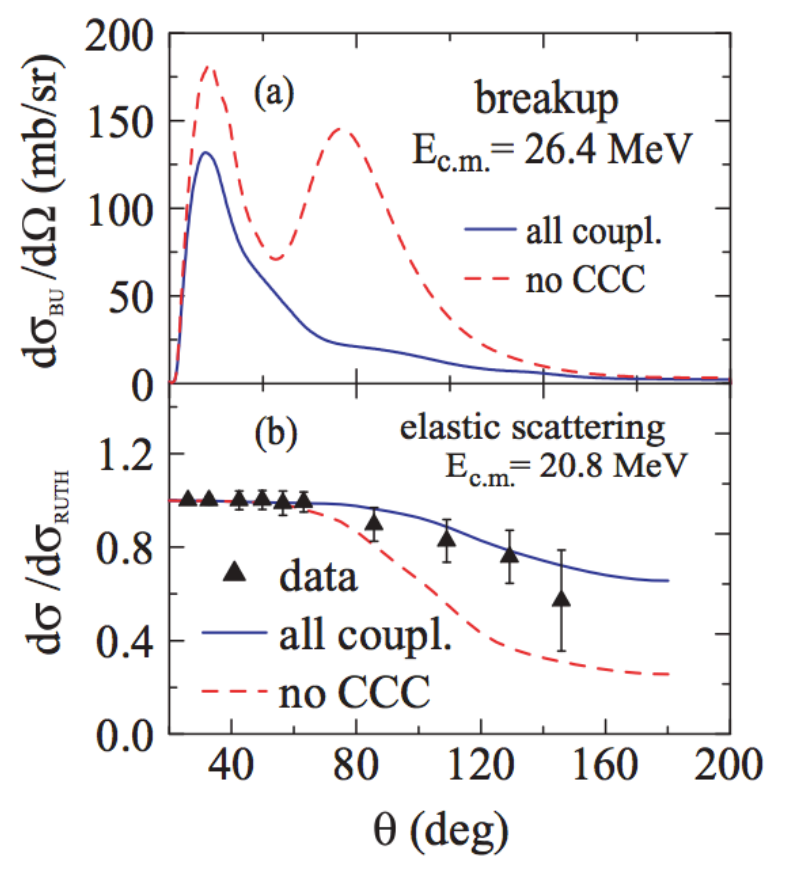

Fig. 9. Breakup (top panel) and elastic (bottom panel) cross sections for the ${ }^{8} \mathrm{~B}+{ }^{58} \mathrm{Ni}$ system, with (solid line) and without (dashed line) CCC. 


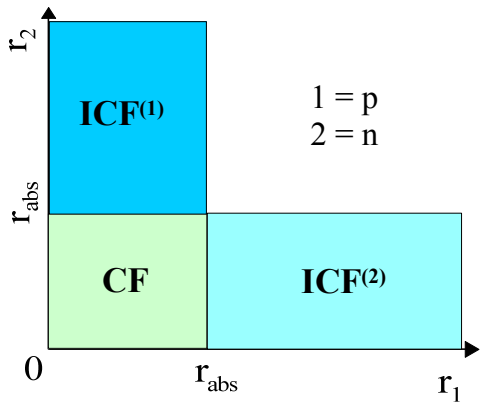

Fig. 10. Integration regions used in Ref. [51].

$\mathbf{r}_{1}, \mathbf{r}_{2}$ the wave function transforms as $\Psi(\mathbf{R}, \mathbf{r}) \rightarrow \Phi\left(\mathbf{r}_{1}, \mathbf{r}_{2}\right)$ and Eq. (2) becomes

$$
\sigma_{\mathrm{F}}=\frac{k}{E} \int d^{3} \mathbf{r}_{1} d^{3} \mathbf{r}_{2}\left|\Phi\left(\mathbf{r}_{1}, \mathbf{r}_{2}\right)\right|^{2} \operatorname{Im}\left\{-V\left(\mathbf{r}_{1}, \mathbf{r}_{2}\right)\right\},
$$

where $V\left(\mathbf{r}_{1}, \mathbf{r}_{2}\right)$ is the sum of complex potentials between the target and each of the fragments. Separate cross sections for CF and ICF of fragments 1 and 2 are obtained by restricting the range of integration over the fragment coordinates, according to figure 10 . This method was used to calculate incomplete fusion of the neutron and the proton following deuteron breakup in the $d+{ }^{7} \mathrm{Li}$ collision. If the modulus of $\mathbf{r}_{i}(i=1,2)$ in the integrand is kept inside the range of absorption, fragment $i$ fuses. Complete fusion is obtained imposing this restriction on coordinates of both fragments, whereas ICF is obtained keeping the coordinates of one of the fragments inside the absorption range and those of the other outside it.

\subsection{Trends of the CF and TF experimental data}

A detailed account of the $\mathrm{CF}$ and TF cross sections of weakly bound systems, together with a list of references about the subject, will be given in Paulo Gomes' talk in this conference. For this reason this issue will be addressed here only briefly.

There are no experimental information about fusion of these systems at extreme sub-barrier energies and most of the data for unstable projectiles below the Coulomb barrier have large error bars. Thus, the experimental information are inconclusive. Sub-barrier fusion data for stable weakly bound projectiles, like ${ }^{6,7} \mathrm{Li}$ and ${ }^{9} \mathrm{Be}$, show some enhancement of the cross section but the enhancement is not very strong. It follows the trend for tightly bound systems, which predicts weak enhancement for systems with small size parameter (for ${ }^{9} \mathrm{Be}+{ }^{208} \mathrm{~Pb}, \zeta \simeq 13$ ).

The most interesting experimental results for weakly bound systems are the CF cross sections for ${ }^{6,7} \mathrm{Li}$ and ${ }^{9} \mathrm{Be}$ projectiles colliding with heavy targets, at above-barrier energies $[57,43,58]$. In such cases, the experimental cross section is suppressed by $\sim 30 \%$, as compared with predictions of optical model calculations with standard potentials. In collisions with medium-heavy targets the suppression is smaller and for light targets there is no effect. On the other hand, the TF cross section does not show noticeable suppression, independently of the target mass. For unstable projectiles, as ${ }^{6} \mathrm{He}$, the situation is different. In this case there is no CF data available. In fact, from the experimental point of view, there is no difference between $\mathrm{CF}$ and $\mathrm{TF}$ when the total charge of the projectile is contained in one of the fragments. However, the TF data for ${ }^{6} \mathrm{He}$ behaves similarly to CF data for stable weakly bound projectiles. One possible interpretation of this fact is that $\sigma_{\text {ICF }}$ for this projectile is much smaller than $\sigma_{\mathrm{CF}}$. A naïve justification for this assumption is that the ${ }^{4} \mathrm{He}$ fragment produced in ${ }^{6} \mathrm{He}$ breakup carries $2 / 3$ of its kinetic energy, while it collides with a higher barrier.

\section{Summary}

We have reviewed some of the main topics in heavy ion fusion reactions, with emphasis in the reaction mechanisms involved. First, we considered fusion of tightly bound systems in three energy regimes: energies a few $\mathrm{MeV}$ above the barrier, energies a few $\mathrm{MeV}$ below and deep sub-barrier energies. We pointed out that channel coupling effects can be relevant above the barrier and lead to strong enhancement at sub-barrier energies. The enhancement increases with the system size. It was argued that this effect is due to the strength of the electromagnetic coupling with collective channels.

The steeper fall off of the fusion cross section at deep sub-barrier energies was then considered and the main theoretical interpretations for this phenomenon have been discussed.

We discussed also the fusion of weakly bound systems, where complete fusion and incomplete fusion may take place. Special attention was given to separate calculations of these fusion processes.

\section{Acknowledgements}

Te authors would like to thank the CNPq, FAPERJ, FAPESP and PRONEX for partial financial support.

\section{References}

1. D. Glas, U. Mosel, Phys. Rev C10, 2620 (1974), see also Nucl. Phys. A237 (1975) 429.

2. C.H. Dasso, S. Landowne, A. Winther, Nucl. Phys. A405, 381 (1983)

3. C.H. Dasso, S. Landowne, A. Winther, Nucl. Phys. A407, 221 (1983)

4. R. Lindsay, N. Rowley, J. Phys. G: Nucl. Part. Phys. 10, 805 (1984)

5. L.F. Canto, P.R.S. Gomes, J. Lubian, L.C. Chamon et al., J. Phys. G: Nucl. Part. Phys. 36, 015109 (2009)

6. L.F. Canto, P.R.S. Gomes, J. Lubian, L.C. Chamon et al., Nucl. Phys. A 821, 51 (2009)

7. C.Y. Wong, Phys. Rev. Lett. 31, 766 (1973)

8. L. Chamon, B. Carlson, L. Gasques, D. Pereira et al., Phys. Rev. C 66, 014610 (2002) 
9. J.R. Leigh, M. Dasgupta, D.J. Hinde, J.C. Mein et al., Phys. Rev. C 52, 3151 (1995)

10. H.Q. Zhang, C.J. Lin, F. Yang, H.M. Jia et al., Phys. Rev. C 82, 054609 (2010)

11. B.B. Back, R.R. Betts, J.E. Gindler, B.D. Wilkins et al., Phys. Rev. C 32, 195 (1985)

12. A. Stefanini, M. Trotta, B.R. Behera, L. Corradi et al., Eur. Phys. J. A 23, 473 (2005)

13. W. Reisdorf, H.F. P., K.D. Hildenbrand, S. Hofmann, G. Münzenberg et al., Nucl. Phys. A 438, 212 (1985)

14. M. Beckerman, M. Salomaa, A. Sperduto, J.D. Molitoris, Phys. Rev. C 25, 837 (1982)

15. M. Beckerman, M. Salomaa, J. Wiggins, Phys. Rev. C 28, 1963 (1983)

16. W. Reisdorf, H.F. P., K.D. Hildenbrand, S. Hofmann, G. Münzenberg et al., Nucl. Phys. A 444, 154 (1985)

17. A. Stefanini, F. Scarlassara, S. Beghini, G. Montagnoli et al., Phys. Rev. C 73, 034606 (2006)

18. D. Ackermann, P. Bednarczyk, L. Corradi, D.R. Napoli et al., Nucl. Phys. A 609, 91 (1996)

19. C.E. Aguiar, V. Barbosa, L.F. Canto, R. Donangelo, Phys. Lett. B 201, 22 (1988)

20. M. Beckerman, J. Ball, H. Enge, M. Salomaa, Phys. Rev. C 23, 1581 (1981)

21. C.L. Jiang, H. Esbensen, K.E. Rehm, B.B. Back et al., Phys. Rev. Lett. 89, 052701 (2002)

22. C.L. Jiang, et al., Phys. Rev. Lett. 93, 012701 (2004)

23. C.L. Jiang, K.E. Rehm, H. Esbensen, R.V.F. Janssens et al., Phys. Rev. C 71, 044613 (2005)

24. M. Dasgupta, D.J. Hinde, A. Diaz-Torres, B. Bouriquet et al., Phys. Rev. Lett. 99, 192701 (2007)

25. A. Stefanini, G. Montagnoli, R. Silvestri, S. Beghini et al., Phys. Rev. C 78, 044607 (2008)

26. C.L. Jiang, B.B. Back, H. Esbensen, J.P. Greene et al., Phys. Rev. C 78, 017601 (2008)

27. A. Stefanini, G. Montagnoli, R. Silvestri, L. Corradi et al., Phys. Letters B 679, 95 (2009)

28. S. Misicu, H. Esbensen, Phys. Rev. C 75, 034606 (2007)

29. C.H. Dasso, G. Pollarolo, Phys. Rev. C 68, 054604 (2003)

30. O. Akyüz, A. Winther, in Nuclear Structure of Heavy Ion Reaction, edited by R.A. Broglia, C.H. Dasso, R.A. Ricci (North Holland, 1981), proc. E. Fermi Summer School of Physics

31. G.R. Satchler, W.G. Love, Phys. Rep. 55, 183 (1979)

32. G.F. Bertsch, J. Borysowicz, H. McManus, W.G. Love, Nucl. Phys. A284, 399 (1977)

33. H. Esbensen, S. Misicu, Phys. Rev. C 76, 054609 (2007)

34. H. Esbensen, C.L. Jiang, A. Stefanini, Phys. Rev. C 82, 054621 (2010)

35. T. Ishikawa, K. Hagino, A. Iwamoto, Phys. Rev. C 75, 057603 (2007)

36. H.J. Krappe, J.R. Nix, A.J. Sierk, Phys. Rev. C 20, 992 (1979)

37. T. Ishikawa, K. Hagino, A. Iwamoto, Phys. Rev. Lett. 103, 202701 (2009)

38. A. Diaz-Torres, D.J. Hinde, M. Dasgupta, C.J. Milburn et al., Phys. Rev. C 78, 064604 (2008)
39. L. Canto, P. Gomes, R. Donangelo, M. Hussein, Phys. Rep. 424, 1 (2006)

40. M.S. Hussein, M.P. Pato, L.F. Canto, R. Donangelo, Phys. Rev. C 46, 377 (1992)

41. N. Takigawa, M. Kuratani, H. Sagawa, Phys. Rev. C 47, R2470 (1993)

42. C.H. Dasso, A. Vitturi, Phys. Rev. C 50, R12 (1994)

43. M. Dasgupta, D.J. Hinde, K. Hagino, S.B. Moraes et al., Phys. Rev. C 66, 041602(R) (2002)

44. K. Hagino, M. Dasgupta, D.J. Hinde, Nucl. Phys. A738, 475 (2004)

45. A. Diaz-Torrez, D.J. Hinde, J.A. Tostevin, M. Dasgupta et al., Phys. Rev. Lett. 98, 152701 (2007)

46. A. Diaz-Torres, J. Phys. G: Nucl. Part. Phys. 37, 075109 (2010)

47. K. Hagino, A. Vitturi, C.H. Dasso, S.M. Lenzi, Phys. Rev. C 61, 037602 (2000)

48. N. Austern, Y. Iseri, M. Kamimura, M. Kawai et al., Phys. Rep. 154, 125 (1987)

49. A. Diaz-Torres, I.J. Thompson, Phys. Rev. C 65 , 024606 (2002)

50. L.F. Canto, J. Lubian, P.R.S. Gomes, M.S. Hussein, Phys. Rev. C 80, 047601 (2009)

51. S. Hashimoto, K. Ogata, S. Chiba, M. Yahiro, Prog. Theor. Phys. 122, 1291 (2009)

52. N. Keeley, K.W. Kemper, K. Rusek, Phys. Rev. C65, 014601 (2001)

53. K. Rusek, N. Keeley, K.W. Kemper, R. Raabe, Phys. Rev. C67, 041604(R) (2003)

54. A. Diaz-Torres, I.J. Thompson, C. Beck, Phys. Rev. C 68, 044607 (2003)

55. C. Beck, N. Keeley, A. Diaz-Torres, Phys. Rev. C 75 , 054605 (2007)

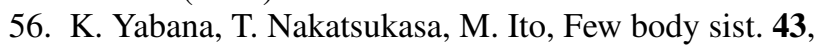
247 (2008)

57. M. Dasgupta, D.J. Hinde, R.D. Butt, R.M. Anjos et al., Phys. Rev. Lett. 82, 1395 (1999)

58. M. Dasgupta, P. Gomes, D. Hinde, S. Moraes et al., Phys. Rev. C 70, 024606 (2004) 